\title{
HOMEOSTASIS MAINTENANCE OF ENCAPSULATED CELLS
}

\author{
Volha Liaudanskaya ${ }^{\#}$, Claudio Migliaresi, Antonella Motta ${ }^{\S}$
}

Department of Industrial Engineering, BIOtech Research Center, University of Trento, via Sommarive 9, 38123 Trento, Italy

European Institute of Excellence on Tissue Engineering and Regenerative medicine, Trento, Italy \#present address: Department of Biomedical Engineering, Tufts University, Medford, MA, USA

\section{${ }^{\S}$ Corresponding author}

\section{ABSTRACT}

Cell niche homeostasis plays a critical role in many bodily functions including tissue functionality, stem cell maintenance and differentiation, wound healing, cancer development and propagation, and many others. Many tissue engineering approaches overlook the importance of engineered constructs homeostasis, in particular for transplantation purposes. Here we present a study of the effect of encapsulation duration on engineered tissue maturation and provide a protocol for the evaluation of critical conditions for transplantation purposes. In brief, SHSY5Y human neuroblastoma cells were encapsulated in $2 \%$ alginate by EHDJ method for up to 4 weeks. We evaluated ECM niche formation and tissue maturation in situ through COL1Al expression. In in vitro conditions we studied the ability of cells to maintain their critical functions after being released from alginate beads. Cellular viability was evaluated via an apoptosis/necrosis detection kit and AlamarBlue assay, and functionality via immunocytochemistry. We proved the importance of engineered tissue homeostasis stabilization for future cell recovery, in particular, for our system cells encapsulated for 28 days met all critical requirements for successful tissue transplantation. Maturation of engineered tissue constructs could be accelerated by enriching alginate with growth factors or ECM molecules.

\section{KEYWORDS}

Homeostasis, Cell encapsulation, EHDJ printing, Organ Printing,

Cell maturation, Alginate, Cell functionality

This article has been accepted for publication and undergone full peer review but has not been through the copyediting, typesetting, pagination and proofreading process which may lead to differences between this version and the Version of Record. Please cite this article as doi: $10.1002 /$ term. 2600 


\section{INTRODUCTION}

"Homeostasis is the capability of biological systems to maintain a relatively stable milieu in a fluctuating external environment" (Kotas and Medzhitov, 2015).

Evolutionary cells of many organisms, including humans, have learned how to survive and function even in extreme conditions due to cell adaptations and homeostasis maintenance. Physiological or pathological stresses can trigger either stress recovery mechanisms or cell death pathways which are strongly correlated with the type and strength of the applied stimulation.

The ability of cells to maintain homeostasis, keeping viability, activity, proliferation and differentiation at a controlled level is critical in all healthy bodily functions including regulation of temperature balance, embryonic development, brain activity, gene expression, but also in pathological conditions such as cancer development and propagation (Kotas and Medzhitov, 2015; Folmes et al., 2012; Mendelson and Frenette, 2014; Stoppato et al., 2012; Daley et al., 2008;). In tissue engineering applications, homeostasis is crucial for transplantation purposes, particularly during transplant adaptation, transplant maturation and extracellular niche formation (Melchels et al., 2011).

Organ printing is a relatively new direction that has received profound attention as a promising technique for the fabrication of cell containing constructs (Mironov et al., 2008; Tabar and Studer, 2014). It consists of a layer by layer 3D deposition of cells that in proper conditions will start forming an in vitro tissue or organ of interest to be later transplanted in vivo, where maturation and remodeling will finally take place. Several methods can be used, one being the use of building blocks of encapsulated cells or tissue spheroids that have demonstrated the ability to fuse together into larger tissue constructs (Liaudanskaya et al., 2015).

Many techniques and protocols were investigated and applied for successful cell encapsulation, such as photolithography, microfluidic encapsulation, ink-jet and bioprinting (Gauvin et al., 2012; Murphy and Atala, 2014; Gasperini et al., 2013; Yao et al., 2009), so as many materials were evaluated for the applications mentioned above (Nicodemus and Bryant, 2008; Gasperini et al., 2014; Das et al., 2015; Lee et al., 2012).

However, the effect of encapsulation on cell maturation was not studied as deeply, and even though some analyses of cell parameters during or post encapsulation have already been done, (Liaudanskaya et al., 2015; Zorlutuna et al., 2012; Mongkoldhumrongkul et al., 2009) there are still many aspects to be addressed for successful tissue fabrication, for example, cell homeostasis and microenvironment maintenance post-encapsulation.

The final goal of cell encapsulation is the formation of homeostatically stable and functional tissue, where the encapsulation matrix would be substituted by the cells' ECM, including growth factors and biomolecules that are important for tissue function. Maturation processes can be considered complete 
when fabricated tissue is able to maintain its physiological structure and stability without external factors and support.

In a previously published study, we presented a detailed analysis on cell viability as well as metabolic activity and stress level of cells encapsulated in alginate beads by electrohydrodynamic jetting (EHDJ) method (Liaudanskaya et al., 2015). Our study showed that cells were capable of recovering from the stress due to the encapsulation process in a short period time, and capable of maintaining their primary functions, like proliferation and ECM production. Moreover, the study indicated that within 3 weeks, cells were able to modify the artificial alginate environment into the functional niche via the production of ECM components like $N$-cadherin.

In the present study we investigated the effect of encapsulation time of SHSY5Y human neuroblastoma cell line (neural progenitors) in alginate gel on the recovery of cell functions after reseeding on tissue culture plates (TCP), as compared to cells just cultured on TCP.

Our focus was to define the requirements necessary for fabricating homeostatically stable cell constructs, which necessitates that encapsulation should not negatively affect cell viability, activity, proliferation, and at last, homeostatic stability. SHSY5Y cell line was used as the model for this study, as suggested by the literature detailing their well-studied stress response and adaptation mechanisms (Ross and Spengler, 2007; Sabirzhanov et al., 2012).

Cells passaged at weeks 12-16 were encapsulated by the EHDJ encapsulation method in $2 \%$ alginate beads (beads size varied around 200 $\mu \mathrm{m}$ ) for up to 4 weeks (Gasperini et al., 2013; Liaudanskaya et al., 2015). Cell functionality and homeostatic stability were evaluated both in vitro and in situ to assess cell behavior post-encapsulation. For in vitro, cells were released from the beads by dissolving the alginate in phosphate-buffered saline (PBS) (without $\mathrm{Ca}^{2+} / \mathrm{Mg}^{2+}$ ) and reseeded on tissue culture

plates (TCP). Un-encapsulated cells seeded on TCP were used as a reference group. At 3 hours, 1, 3, $5,7,14,21$ and 28 days after encapsulation, cells were subjected to a set of tests. Cell viability was assessed with apoptotic/necrotic detection kit, metabolic activity with Alamar blue assay, and proliferation level with DNA quantification assay. Adhesion, motion, and cell-cell communication were evaluated at days $1,3,5$ and 7 via quantification of $N$-cadherin expression. In situ tests were done on cells in the beads in order to prove niche formation and homeostatic stabilization. More specifically, collagenl expression was evaluated via quantitative reverse transcription polymerase chain reaction in real time (RT-qPCR), and cells imaged within 4 weeks of encapsulation to evaluate any morphological changes.

\section{MATERIALS AND METHODS}

Alginic acid sodium salt from brown algae and calcium chloride dehydrate was purchased from Sigma-Aldrich (USA). Phosphate-buffered saline without calcium/magnesium (PBS), 
Dulbecco's modified eagle medium (DMEM), Quant-iT PicoGreen dsDNA Assay, and 4',6Diamidino-2-Phenylindole, Dihydrochloride (DAPI) were obtained from Invitrogen (USA). The encapsulation system consisted of a generator ES30 (Gamma High Voltage Research Inc., USA), a pump (NE-300, New Era Pump Systems, USA), a polyetrafluorethylene tube and a gauge 33 stainless steel needle (outer diameter: $0.210 \mathrm{~mm}$, inner diameter: $0.108 \mathrm{~mm}$ Hamilton, Bonaduz/Switzerland). SHSY5Y human neuroblastoma cell line (ATCC ${ }^{\circledR}$ CRL$2266^{\mathrm{TM}}$, UK) was given as a courtesy by CIBIO Research center at University of Trento (Italy). To permeabize cells, sonication (UP400S Heilscher, Germany) was used. At last, apoptotic/necrotic kit was purchased from PromoKine (PK-CA707-30017) and N-cadherin antibody from Santa Cruz (rabbit sc-7939).

\section{Hydrogel preparation}

Sodium alginate powder was dissolved in PBS (without calcium and magnesium) at room temperature under mild stirring conditions for 6-8 hours, at a final concentration of $2 \%$ $(2 \mathrm{~g} / 100 \mathrm{ml})$. The solution was sterilized through a $0.22 \mu \mathrm{m}$ filter at a flow rate of $5 \mu \mathrm{m} / \mathrm{min}$ in a sterile biological cabinet. The sterile alginate solution was used for cell encapsulation within 24 hours of filtration.

\section{Cell culture}

The SHSY5Y human neuroblastoma cell line was subcultured in T25-T175 tissue culture flasks as a monolayer (passages $12-16$ ) at $37^{\circ} \mathrm{C}$ under $5 \% \mathrm{CO}_{2}$ in high glucose Dulbecco's Modified Essential Medium (DMEM) with 10\% FBS (Euroclone), 2mM Glutamine and 1\% penicillin-streptomycin mixture (Sigma, USA). Medium was changed every third day. The cells were passaged till $90-95 \%$ confluency before encapsulation.

\section{Preparation of alginate-cell suspension}

Confluent cell monolayers were detached by trypsinization, cells were collected in a $15 \mathrm{ml}$ vial, centrifuged at $1000 \mathrm{rpm}$ for 5 minutes, and the supernatant removed. The cell pellet was then resuspended in PBS, and centrifuged one more time in order to remove any medium residues containing cations capable of triggering alginate gelation. Finally, sodium alginate solution was added to obtain a suspension of $5 \mathrm{mln}$ cells per $1 \mathrm{ml}$ of alginate. Cell density prior to alginate addition was estimated by Cellometer Auto T4 (Nexcelom, USA), using Trypan Blue 0,4\% (Life technologies, USA) as contrast agent. 


\section{Cell encapsulation by electrohydrodynamic jet method (EHDJ)}

Cell encapsulation was performed following a previously published approach (Gasperini et al., 2013). In brief, the sterilized encapsulation set-up was placed under a biological hood, and consisted of a 3 $\mathrm{ml}$ syringe loaded with the cell-alginate suspension placed on a pump, a vertically placed stainless steel needle connected with a polytetrafluoroethylene tube to the syringe pump, a metallic plate $5 \mathrm{~cm}$ distant from the needle, and a Petri dish filled with the calcium chloride solution placed below the needle on the plate. The needle and metallic plate were connected to the positive and negative pole of a voltage generator accordingly.

Once a solution flux reading of $0.05 \mathrm{ml} / \mathrm{min}$ was established and the generator switched on $(\mathrm{V}=8 \mathrm{kV})$, droplets of alginate solution containing cells were ejected from the needle directly into a $400 \mathrm{mM}$ $\mathrm{CaCl}_{2}$ bath. After 10 minutes the beads were collected, washed three times with complete DMEM, and placed in 6-well tissue culture plates in full media for incubation until the experimental time points.

\section{Cell release}

For in vitro evaluations, cells were released from the beads and reseeded onto 12 -well tissue culture plates in a density of 100,000 cells/well, consistent for all experimental groups, including the control group of cells (that were not encapsulated in the beads). In brief, the alginate beads were dissolved by removing the DMEM media containing $\mathrm{Ca}^{2+}$ cations, followed by a short wash in PBS at room temperature and 15 minutes of incubation in PBS (without $\mathrm{Ca}^{2+} / \mathrm{Mg}^{2+}$ ) at $37^{\circ} \mathrm{C}$ to trigger the ion exchange with the surrounding buffer. To ensure complete alginate dissolution, samples were gently vortexed every $5 \mathrm{~min}$ until the cell suspension was homogeneous without cell clusters. After, the supernatant was centrifuged and the cell pellet resuspended in $5 \mathrm{ml}$ of full media for counting. An aliquot of the solution was taken for counting with a Cellometer Auto T4 (Nexcelom, USA), using Trypan Blue $0.4 \%$ (Life technologies, USA) as contrast agent.

\section{In vitro evaluation}

All assays were performed in either in situ or in vitro conditions. For in situ, alginate beads containing cells were collected for analysis at days 1, 7, 14, 21 and 28, whereas for in vitro studies, cells were released from the beads and reseeded in $2 \mathrm{D}$ conditions, 3 hours, 1, 3, 5, 7, 14, 21 and 28 days after encapsulation. All experimental groups were compared to cells grown on tissue culture plates for up to 7 days. All experiments were conducted in triplicate.

Microscopy bright field images

Cell morphology and migration were evaluated with a bright field inverted Nikon Axiovert microscope at the established experimental time points mentioned above. 


\section{Cell viability}

Cell viability was evaluated using PromoKine apoptotic/necrotic kit. Briefly, cells were fixed with $4 \%$ PFA on the tissue culture plate at the established time points. Samples were washed twice with $1 \mathrm{X}$ Binding buffer and then incubated for $15 \mathrm{~min}$ in a solution containing $5 \mu \mathrm{l}$ of FITC-Annexin $\mathrm{V}$ and $5 \mu \mathrm{l}$ of Ethidium Homodimer III in $100 \mu \mathrm{l}$ of $1 \mathrm{X}$ Binding Buffer (light protected). Next, samples were washed twice with $1 \mathrm{X}$ Binding buffer and stained for $5 \mathrm{~min}$ with DAPI (light protected). After a brief washing in PBS images were taken with a Nikon (A1 model) confocal microscope. Image analyses were performed with the ImageJ software cell counter plugin, where cells positive for Annexin V (green signal) were counted as apoptotic, cells positive for Ethidium Homodimer III (red signal) were counted as necrotic, and total cell number obtained by counting DAPI (blue signal) positive cells (FigureS1 represents images taken after staining with Apoptotic/Necrotic kit). Data were normalized against the total cell count of DAPI positive cells. Collected data were analyzed with GraphPrism 6 software.

\section{Cell proliferation}

Cell proliferation was evaluated using the PicoGreen assay. In brief, cells were released from alginate beads as previously described, and $0.05 \%$ Triton-X solution was added for DNA isolation, followed by sonication for 10 seconds (cycle: 1, amplitude 40\%). DNA extract was diluted to the test sensitive concentration and PicoGreen reagent used for quantification. The fluorescence intensity of PicoGreen - DNA complexes was measured in 96-well black plates using a plate reader (485 nm excitation and $538 \mathrm{~nm}$ emission; Safire, Tecan, Austria). To correlate fluorescence intensity with DNA concentration, a calibration curve was generated using the DNA standards provided with the assay.

\section{Cell activity}

Cell activity includes all biological processes, such as oxidative response, oxygen utilization, cell energy production, cell metabolism, and many protein functions. In the current study, cell metabolic activity was measured with an AlamarBlue ${ }^{\circledR}$ assay. Cells were incubated in the dark with $10 \%$ AlamarBlue solution in full DMEM media for 2.5 hours, then $100 \mu \mathrm{l}$ of AlamarBlue/Media mixed solution was transferred from each sample to the new 96-well plate and fluorescence signals measured with a plate reader (485 nm excitation and $538 \mathrm{~nm}$ emission; Safire, Tecan, Austria). Medium without cells was used to determine absorbance of negative control.

\section{Cellular communication}

To examine cell-cell communication, N-cadherin production was analysed via immunocytochemistry assay. Samples were fixed with 4\% PFA for $30 \mathrm{~min}$ and then permeabilized in a blocking buffer composed of $1 \%$ BSA and $0.3 \%$ TritonX in PBS for 1 hour. Samples were incubated with a primary anti-N-cadherin antibody (sc-7939) overnight at $4 \mathrm{C}^{\circ}$ and washed after 
incubation 3 times in PBS for 10 minutes. At last, a secondary anti-rabbit 488 antibody was applied for 1 hour at room temperature followed by a triple wash in PBS. Cell nuclei were counterstained with DAPI solution for $5 \mathrm{~min}$, and washed twice with PBS. Results were evaluated with the Nikon confocal microscope.

\section{Quantitative reverse transcription polymerase chain reaction in real time (RT-qPCR)}

Gene expression was evaluated by RT-qPCR assay. Briefly, cells were released from the beads (as described above) and mRNA isolated using QIAGEN RNA extraction kit following the manufacturer protocol. Total RNA was resuspended in RNAse-free water and final concentration was measured with standard spectrophotometry. Reverse transcription was performed in a final volume of $20 \mu 1$ with $1 \mu \mathrm{g}$ of RNA being used for transcription (SuperScript III Reverse Transcriptase, Invitrogen, UK). Transcribed cDNA was diluted 20 times for further PCR analysis. GAPDH and COL1A1 primers were designed using Beacon Designer software, and the sequence for RPS18 primers taken from PrimerBank (Spandidos et al., 2010). COL1A1_f: 5'-CCACCAATCACCTGCGTACAGAA-3'; COL1A1 r : 5'-GGGCAGTTCTTGGTCTCGTCAC-3'; GAPDH f: 5'-TCGGAGTGAACGGATTTC-

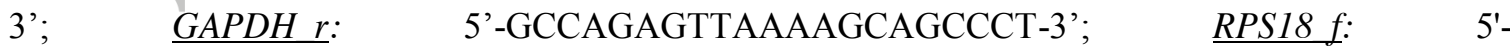
ATCACCATTATGCAGAATCCACG-3'; $\underline{\text { RPS18 } r}:$ : 5'-GACCTGGCTGTATTTTCCATCC-3'. Reactions were conducted in $10 \mu 1$ volumes: $2 \mu 1 \mathrm{cDNA}, 0.2 \mu 1$ of each primer, $5 \mu 1 \mathrm{KAPA}$ SYBR GREEN, and 2.6 $\mu$ l of RNA-free water. Amplification and detection were carried out using CFX96

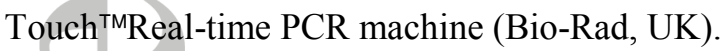

\section{Statistical analysis}

All results were analyzed with GraphPad Prism 6 software. Statistical analysis for more than two groups was conducted using a one-way ANOVA with Tukey's post-hoc test. A p-value of less than 0.05 was considered statistically significant. Gene expression analysis was performed using the $\mathrm{ddC}_{\mathrm{t}}$ approach, using the RPS18 gene as a reference. Expression of the targeted genes was compared to the control group of SHSY5Y cells grown on tissue culture plates. All assays were performed in triplicate.

\section{RESULTS}

\section{In situ evaluation of cell adaptation to artificial microenvironment}

Cell behavior within alginate beads was monitored daily with a brightfield inverted microscope, and images taken to visualize differences in cell behavior and modifications in microenviroment. Cell viability and proliferation parameters in situ were analysed in detail in a previously published study (Liaudanskaya et al., 2015), thus, cell behavior was analysed in terms of homeostasis maintenance and ability to remodel alginate to their funtional niche. Figure 1A summarizes cell behavior cultured 
in alginate beads up to 4 weeks of encapsulation. An increase in cell number was observed starting three weeks post-encapsulation. At the same time, it was observed that beads coalesce and that cells communicate between neighbouring beads. This phenomenon was even more evident at day 28, where the profile of the alginate beads started to dissolve. The reported morphological pattern is in agreement with the results of Figure 1B of the RT-qPCR assay for collagen1 (COL1A1) expression. The control represents collagen 1 expression for the reference cell group, i.e., cells cultured on TCP till $85-90 \%$ conluency. Collagen 1 expression was down-regulated within 3 days post-encapsulation, significantly increased up to day 14 , and then gradually down-regulated until the last experimental time point.

Figures 2-4 represent viability, activity and proliferation of cells not encapsulated before taken as a control and of cells released from the beads after different encapsulation times and cultivated on TCP for different times, as indicated. In particular, in Figure 2 data regarding cell death, total amount of cells adhered to the surface of TCP, total amount of cells in suspension, and cell metabolic activity per cell at days 1 and 7 are reported and compared with the values of the control. Figures 3 and 4 are bright-field images of the reseeded cells and confocal microscopy images of the immunocytochemistry assay against N-cadherin, respectively. Supplementary figures S2-S5 contain the full data set for figure 2, with additional time points at Days 3 and 5.

From day 1 to day 7 the control group generally showed a low level of cell death, with decreasing levels of necrosis up until the last experimental time point (Fig. 2A), as well as high proliferation and activity level (Fig. 2B). Moreover, the images in Figs. 3 and 4 indicate a homogeneous cell population with epithelial like morphology, as is specific for the SHSY5Y cell line, and an increase in the level of $\mathrm{N}$-cadherin expression.

Cells released 3 hours after encapsulation behaved statistically identical as compared to the control group. The general cell death rate was on the same level, with a slight increase in necrosis on the third day of culture, and a similar number of cells adhered to the tissue culture plates and proliferated in the same pattern as the control group. A significant difference in the number of cells in suspension at day 7 after reseeding was found. At last, no differences were observed in the cell phenotypes and growth patterns, so as in the level of $\mathrm{N}$-cadherin expression.

Cells reseeded one day after encapsulation displayed a significant increase in cell death rate (both apoptotic and necrotic) after 1 day of culture, which then decreased towards the $7^{\text {th }}$ day. Cell proliferation significantly slowed down, but reached the control level by the $7^{\text {th }}$ day of culture. At last, cell-cell communication was disturbed, as indicated by the decreased amount of $\mathrm{N}$-cadherin expression, though morphologically cells were similar to the control group (Figure 4).

Experiments on cells encapsulated for 3,5 and 7 days showed a slower proliferation rate than the control and an increase in the level of cell death, but only at the first and the last days of culture. The 
3-days group had the highest amount of cells in suspension at day 7 after reseeding, and the lowest level of $\mathrm{N}$-cadherin expression at this time. The 5-days group had a significantly lower amount of cells in suspension by the $7^{\text {th }}$ day of culture, and a very high level of $\mathrm{N}$-cadherin expression. At last, cultures on cells encapsulated for 7 days had the highest number of cells in suspension the first day after reseeding with a very low level of N-cadherin expression at this time point; however, at day 7 , values became similar to those of the control. A shift to mesenchymal-like morphology in the 5 days and 7 days groups was observed, but the growth pattern was the same as in the control group, with a homogeneous distribution on the surface of the tissue culture plate (Figure 4).

Cells encapsulated in the alginate beads for 14 days presented the lowest level of necrosis the first day after reseeding, but the highest apoptotic level among all experimental groups and samples, which completely recovered to the control level towards the $7^{\text {th }}$ day of culture. The total cell number was relatively low, but higher than in the 3-7 days groups. A very high number of cells were in suspension the first day (supports the high amount of apoptotic cells), but the amount of cells in suspension returned to the control level at the next experimental time point. The activity of this culture group was relatively low as well, however this group had the highest level of $\mathrm{N}$-cadherin expression out of all experimental time points. At last, these cells had mesenchymal like morphology and started to grow in tighter groups (Figures 2-4).

Cells encapsulated for 21 days presented the highest level of necrotic cell death, that gradually decreased towards the last experimental day. The apoptotic cell death level was elevated as well the first day after reseeding, but completely recovered towards the last day of culture. This group also had a relatively low proliferation level, low activity, and presented cells that assembled in tight clusters with very high level of $\mathrm{N}$-cadherin expression, assuming a mesenchymal-like phenotype (Figures 24).

Cells encapsulated for 28 days in the beads had the highest proliferation rate, and a very high activity level. A very high number of cells in suspension was observed the first day after reseeding, with the highest level of $\mathrm{N}$-cadherin expression of all experimental points, and with cells growing in very tight groups in multi-layers with overexpression of $\mathrm{N}$-cadherin in these areas (Figures 2-4).

\section{DISCUSSION}

Homeostatically stable engineered tissue should maintain its specific functions in fluctuating external conditions (Folmes et al., 2012; Mendelson and Frenette, 2014) and preserve cell viability, activity and communication capability (Daley et al., 2008; Lub et al., 2011; Chen et al., 2006; Tbar and Stider, 2014). Homeostasis maintenance of cell constructs is critical for tissue maturation, integration and adaptation post-encapsulation, as sketched in Figure 5. In this paper we have evaluated cell homeostasis after encapsulation in $2 \%$ alginate beads for 3 hours up to 4 weeks. 
The first and most crucial parameters for engineered tissue are stress response and cell viability after encapsulation. The presence of dead cells in fabricated structures may trigger an apoptotic cascade or necrotic core formation (Sabirzhanov et al., 2012; Kalluri and Zeisberg, 2006). Early death rate is strictly controlled for in all tissue engineering methods, however, late time death propagation due to hypoxia or starvation has to be controlled as well.

In previously published work we presented results of low death rate and absence of hypoxia-necrosis propagation within 4 weeks of encapsulation (Liaudanskaya et al., 2015). Here, we observed that cells cultured in the beads from one up to 21 days, released and reseeded in control conditions, had a significantly up-regulated cell death rate, whereas cells cultured for 28 days in the beads had a death rate comparable to the reference group. These data indicate the engineered construct is not mature 3 weeks after encapsulation, and upon transplantation, could trigger a severe immune response, fibrosis and finally rejection after implantation.

The next parameters are cell activity and proliferation. Cell number and activity are specific for every tissue, and are critical for building the ECM, promoting self-support after artificial matrix degradation, and assisting in integration post-implantation. Expansion protocols can be complex for many cell types, especially those derived from patients with genotypically damaged cells, or elderly people whose cells have a lower capacity of self-renewal and proliferation, thus, homeostatic stability will be critical to reach the proper proliferation and activity rate for these cells especially. Factors such as oxygen, nutrient, and growth factor supply, as well as waste removal have to be constantly controlled (for this particular requirement, bioreactors with constant flow will be the most favorable). We found (Figure 2B, 2D) that the proliferation and activity of cells cultured in the alginate beads for up to 21 day was highly down-regulated, however, significant up-regulation of proliferation was observed in the cells cultured in the beads for 28 days. Metabolic activity data normalized per cell number (Fig. 2D) don't display a regular trend with fluctuations generally indicating a reverse relationship between cell number and cell metabolic activity. However, metabolic activities per cell at 7 days culture for all encapsulation times are comparable or higher than control. The mechanistic links between cellular metabolism and growth control are not fully understood (Vander Heiden et al., 2009). Interestingly, as shown in Fig. 5S, considering the whole cell population of the different systems evolved from the same initial cell number, metabolic activity shows an increase with time of culture, particularly relevant for cell encapsulated for 28 days.

We hypothesized that cells were recovering during the first week after encapsulation, followed by 2 weeks of alginate remodeling, and when the environment was supposedly adapted (ECM components produced and organized), cells started to proliferate and populate the surrounding environment (Figures 3-4). 
At last, the major requirement aimed at improving post-transplantation adaptation is the ability of cells to assemble into complex 3D structures, communicate, migrate and form functional niches (Figure 5). Homeostasis and niche regulation were studied in depth in the stem cell field due to their importance in self-renewal and differentiation processes (Jones and Wagers, 2008; Scadden, 2006; Mendeklson and Frenette, 2014; Wagers, 2012; Chen et al., 2013). Similar to other cell types, through communication and contact, they are capable of maintaining proper functionality or, for instance, triggering an apoptotic cascade in a matter of hours. Initially, to check niche formation and cell adaptation in it, we evaluated cell behavior in the beads and their ability to produce components of ECM through collagenlal expression (Figure 1). The first week after encapsulation expression of COL1A1 progressively decreased (stress recovery time), but after 5 days started to grow until day 21 when the niche was supposedly formed. At this time, cells started to display normal activity and growth, which supports the previously described hypothesis. However, as it was mentioned, the matrix has to be degraded and cells have to be capable of maintaining communication on their own. To evaluate cell migration, communication and self-assembly capacity, cells encapsulated for 3 hours up to 4 weeks were released from the alginate beads and cultured on TCP. Bright field images of cell morphological changes (Figure 3), and confocal images of immunocytochemistry against the ECM component proteoglycan $\mathrm{N}$-cadherin (Figure 4) were collected. Reseeded cells from all samples quickly adhered to the TCP surfaces, morphologically assuming a spindle shape, probably induced by phenotypic changes. Interestingly, cells re-seeded after 28 days of encapsulation showed higher proliferation and more cell-cell contact (Figure 3-I). Cell adhesion and cell interconnections were confirmed by confocal images on N-cadherin stained samples (Figure 4). All samples showed an upregulation of $\mathrm{N}$-cadherin from day 1 to day 7 , however, it was confirmed that cells encapsulated for 28 days increase their interconnectivities mediated by $\mathrm{N}$-cadherin synthesis. Due to defined connectivity by formation of contacts between individual cells, a higher cell proliferation and migratory potential was seen in particular at day 7 . These observations are in agreement with the collagen type I expression analysis performed on cells encapsulated and cultured up to 4 weeks (Figure 1B). It was evident that the ability of cells to build their surrounding matrix, producing a network of collagen able to control interconnectivity among cells in 3 dimensions, was particularly effective in the 28 days group. It should be noted that cell-cell contact occurring in the microenvironment provides determinant cues for differentiation, assembling, and communication with surrounding tissues thus driving integration post-implantation. The last 2 groups of cells encapsulated for 21 and 28 days, respectively, showed the accepted behavior for this requirement: high selfassembly and $\mathrm{N}$-cadherin expression. However, considering all the other requirements, only the 28 days group can be proposed as optimal for transplantation as this group met all the requirements for homeostasis stabilization in the fabricated construct including high viability, activity, proliferation and $3 \mathrm{D}$ tissue mimicking behavior. 
Even though the only group to meet all the described requirements for homeostatic stabilization was the group of cells encapsulated for 28 days, the group of cells encapsulated for 21 days fully recovered from the encapsulation stress, with a major difference in proliferation rate.

\section{CONCLUSIONS}

For successful 3D tissue fabrication, cell homeostasis and niche formation have be to deeply studied and controlled for every tissue engineering method. This paper reviews several requirements necessary to control homeostatic stability, such as viability, activity, proliferation, and adaptation to the surrounding environment. Implementation of these requirements in tissue engineering can significantly improve post-implantation adaption and recovery, so as decrease the risk of immune rejection and fibrosis.

\section{Acknowledgments}

Authors acknowledge Alexander N Berk, BME undergraduate student at Tufts University for English grammar correction, moreover the Centre of Integrative Biology (CIBIO) of University of Trento for providing SHSY5Y cell line and opportunity to use RT-PCR equipment for some assays under the supervision of Dr. Antonio Casini.

Conflict of interest

No competing financial interests exist.

References

Chen S, Lewallen M, Xie T. 2013, Adhesion in the stem cell niche: biological roles and regulation, Development, 2:255-265

Daley WP, Peters SB, Larsen M. 2008, Extracellular matrix dynamics in development and regenerative medicine, J Cell Sci, 121:255-264.

Das S, Pati F, Choi YJ, Rijal G, Shim JH, Kim SW, Ray AR, Cho DW, Ghosh S. 2015,

Bioprintable, cell-laden silk fibroin-gelatin hydrogel supporting multilineage differentiation of stem cells for fabrication of three-dimensional tissue constructs, Acta Biomaterialia, 11:233- 
Folmes CDL, Dzeja PP, Neslon TJ, Terzic A. 2012, Metabolic plasticity in stem cell

homeostasis and differentiation, Cell: Stem Cell, 11:596-606

Gasperini L, Maniglio D, Migliaresi C. 2013, Microencapsulation of cells in alginate through an electrohydrodynamic process, J Bioact Compat Pol, 28:413-423

Gasperini L, Mano JF, Reis RL. 2014, Natural polymers for the microencapsulation of cells,

$J$ R Soc Interface, 11:1-19

Gauvin R, Chen YC, Lee JW, Soman P, Zorlutuna P, Nichol JW, Bae H, Chen S, Khademhosseini A. 2012, Microfabrication of complex porous tissue engineering scaffolds using 3D projection stereolithography, Biomaterials, 33:3824-3834

Gurkan UA, Fan Y, Xu F, Erkmen B, Urkac ES, Parlakgul G, Bernstein J, Xing W, Boyden ES, Demirci U.2012, Simple precision creation of digitally specified, spatially

heterogeneous, engineered tissue architectures, Adv Mater, 8:1192-1198

Heden P, Sellman G, von Wachenfeldt M, Olenius M, Fagrell D. 2009, Body shaping and volume restoration: the role of hyaluronic acid, Aesthet Plast Surg, 33:274-282

Hynes RO. 2009, The extracellular matrix: not just pretty fibrils, Science, 326:1216-1219

Jones DL, Wagers AJ. 2008, No place like home: anatomy and function of the stem cell niche, Nat Rev Mol Cell Biol, 9:11- 21

Kalluri R, Zeisberg M. 2006, Fibroblasts in cancer, Nature Reviews, 6:392-4011 
Kotas ME, Medzhitov R. 2015, Homeostasis, Inflammation and disease susceptibility, Cell, 160:816827

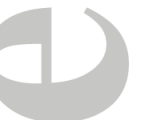

Lee KY, Moone, DJ. 2012, Alginate: properties and biomedical applications, Prog Polym Sci, 37:106126

Li Z, Kawashita M. 2011, Current progress in inorganic artificial biomaterials, J Artif Organs, 14: 163-170

Liaudanskaya V, Gasperini L, Maniglio D, Motta A, Migliaresi C. 2015, Assessing the impact of electrohydrodynamic jetting on encapsulated cell viability, proliferation, and ability to selfassemble in three-dimensional structures, Tissue Eng. Part C, 21:631-638

Lu P, Takai K, Weaver VM, Werb Z. 2011, Extracellular matrix degradation and remodeling in development and disease, Cold Spring Harbor Perspect Biol, 3(12):1-24

Melchels FJ, Malda N, Fedorovich J, Alblas J, Woodfield T. 2011, Organ Printing, Comprehensive Biomaterials, ed Ducheyne P, Elsevier Ltd., Amsterdam, The Netherlands, 587-606

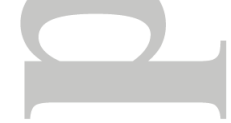

Mendelson A, Frenette PS 2014, Hematopoietic stem cell niche maintenance during homeostasis and regeneration, Nat Med, 20:833-846.

Mironov V, Kasyanow V, Drake C, Marward R. 2008, Organ printing: promises and challenges, Future Medicine, 3:93-103

Mongkoldhumrongkul N, Best S, Aarons E, Jayasinghe SN. 2009, Bio-electrospraying whole human blood : analysing cellular viability at a molecular level, J Tissue Eng Regen Med, 
Murphy SV, Atala A. 2014, 3D bioprinting of tissues and organs, Nat Biotechnol, 32:773-785

Murphy SV, Skardal A, Atala A. 2013, Evaluation of hydrogels for bio-printing applications, $J$

Biomed Mater Res Part A, 101:272-284

Nicodemus GD, Bryant SJ. 2008, Cell encapsulation in biodegradable hydrogels for tissue engineering applications, Tissue Eng Part B, 14:149-165

Ross RA, Spengler BA. 2007, Human neuroblastoma stem cells, Semin Cancer Biol, 17:241-247

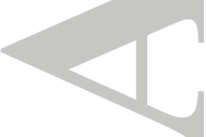

Sabirzhanov B, Stoica B A, Hanscom M, Piao CS, Faden A I. 2012, Over-expression of HSP70 attenuates caspase-dependent and caspase-independent pathways and inhibits neuronal apoptosis, $J$

Neurochem, 123:542-554

Scadden DT. 2006, The stem cell niche as an entity of action, Nature, 441:1075-1079

Spandidos A, Wang X, Wang H, Seed B. 2010, PrimerBank: a resource of human and mouse PCR primer pairs for gene expression detection and quantification, Nucleic Acids Res, 38:792-799

Stoppato M, Carletti E, Sidarovich V, Quattrone A, Unger RE, Kirkpatrick CJ, Migliaresi C, Motta A. 2012, Influence of scaffold pore size on collagen I development: A new in vitro evaluation perspective, J Bioact Compat Pol, 28:16-32

Tabar V, Studer L. 2014, Pluripotent stem cells in regenerative medicine: challenges and recent progress, Nat Genet, 15:82-92

Vander Heiden MG, Cantley LC, Thompson CB. 2009, Understanding the Warburg Effect: The Metabolic Requirements of Cell Proliferation, Science, 324 (5930): 1029-1033 
Wagers AJ. 2012, The stem cell niche in regenerative medicine, Cell: Stem cell, 10:362-369

Whitesides GM. 2006, The origins and the future of microfluidics, Nature, 442:368-373

Yao R, Zhang R, Wang X. 2009, Design and evaluation of a cell microencapsulating device for cell assembly technology, J Bioact Compat Pol, 24:48-56

Zorlutuna P, Annabi N, Camci-Unal G, Nikkhah M, Cha JM, Nichol JW, Manbachi A, Chen S, Khademhosseini A. 2012, Microfabricated biomaterials for engineering 3D tissues, Adv Mater, 24:1782-1804

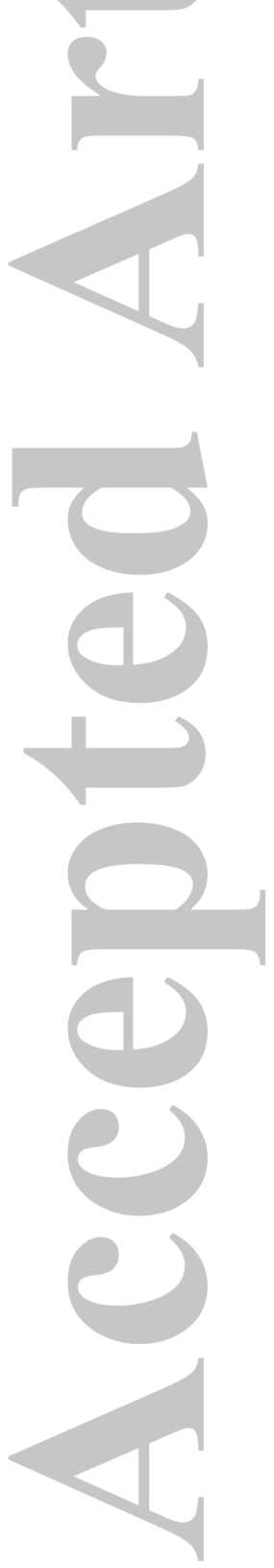




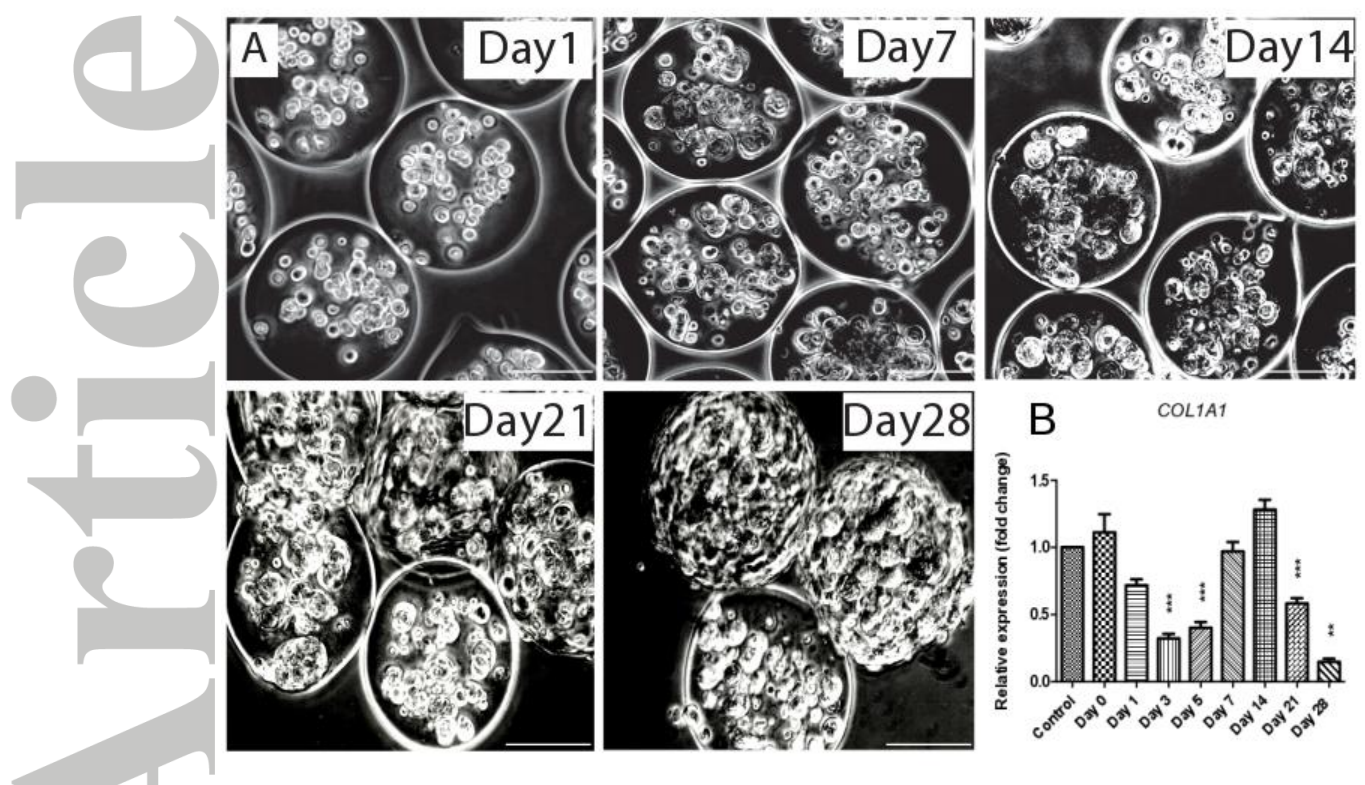

Figure 1. Cells adapt the artificial environment to their functional niche after 3 weeks of encapsulation. A)Brightfield images of SHSY5Y cells encapsulated in $2 \%$ alginate beads by EHDJ method up to 4 weeks; B) RT-qPCR assay of ECM marker - collagen 1A1 experession in 8 studying and control groups. Scale bar: $100 \mu \mathrm{m}$. All experimental groups were compared to the control and $*_{p}$ value less than 0.05 was considered significant. All experimental groups were prepared in triplicates. Control represents collagen 1 expression for the reference group.

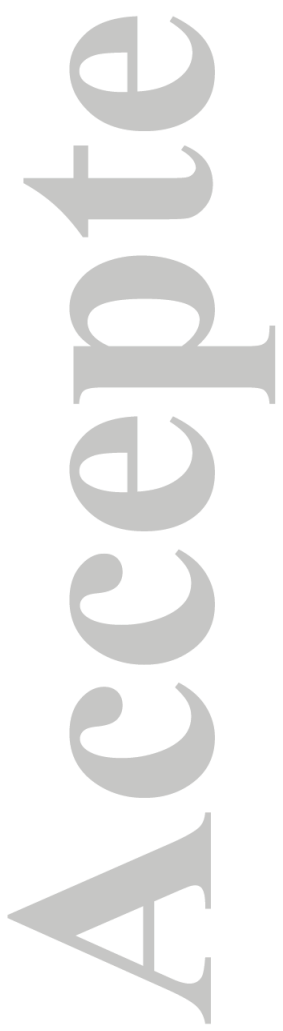



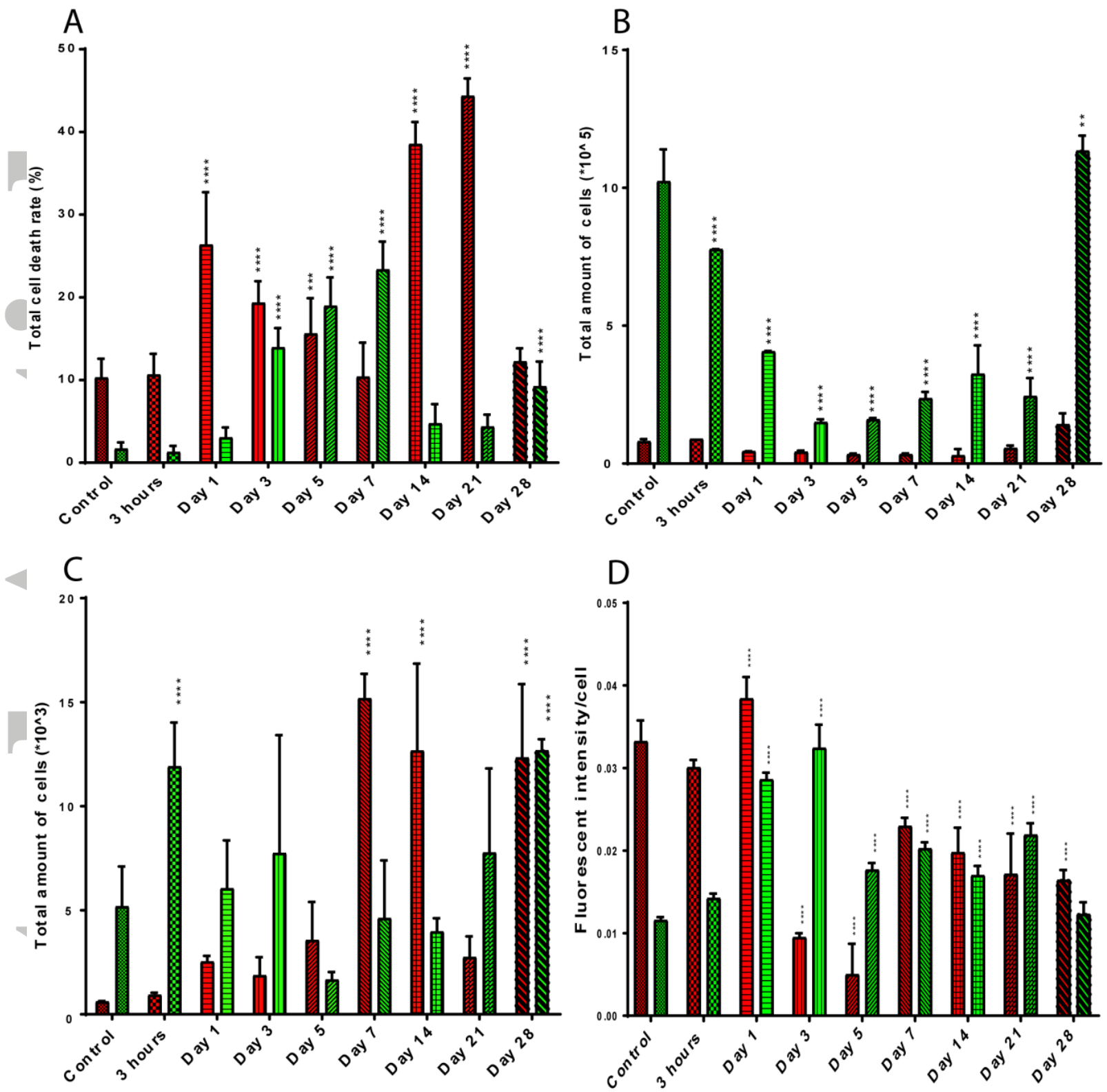

Time points of the conducted in vitro assays

$$
\text { - Day } 1 \square \text {-Day } 7
$$

Figure 2. Cells encapsulated for 4 weeks and reseeded on TCP have the lowest death rate, and recovered proliferation rate towards the control group. Statistical analysis of SHSY5Y viability, activity and proliferation after encapsulation with EHDJ system in $2 \%$ alginate beads and incubation in full media for 3 hours up to 4 weeks at $37^{\circ} \mathrm{C}$ in $5 \% \mathrm{CO}_{2}$ incubator, followed by releasing and reseeding on tissue culture plates. After cells were reseeded they were subjected to a set of tests at 4 experimental time points: $1,3,5$ and 7 days. A) Total cell death rate was measured with Apoptotic/Necrotic kit; B) Total amount of cells adhered to the surface of TCP and C) Total amount of cells in suspension was measured with PicoGreen assay; D) Cell metabolic activity was measured with AlamarBlue assay. In full all the graphs presented in the supplementary document. All experimental groups were compared to the control group and ${ }^{*} p$ value less than 0.05 was considered significant. All experimental groups were prepared in biological triplicates. 


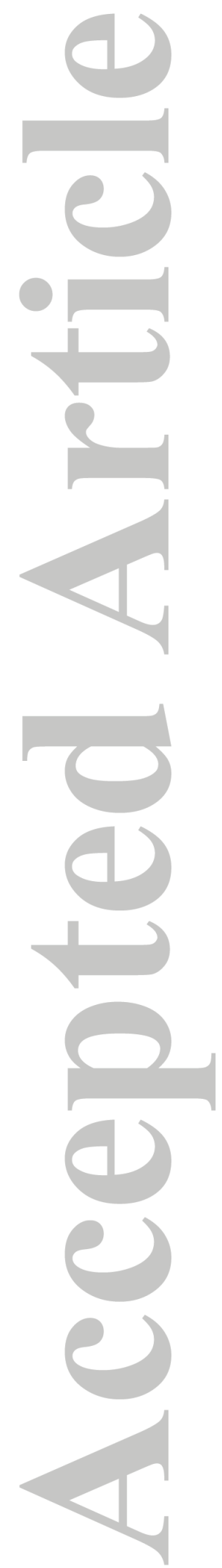

This article is protected by copyright. All rights reserved. 


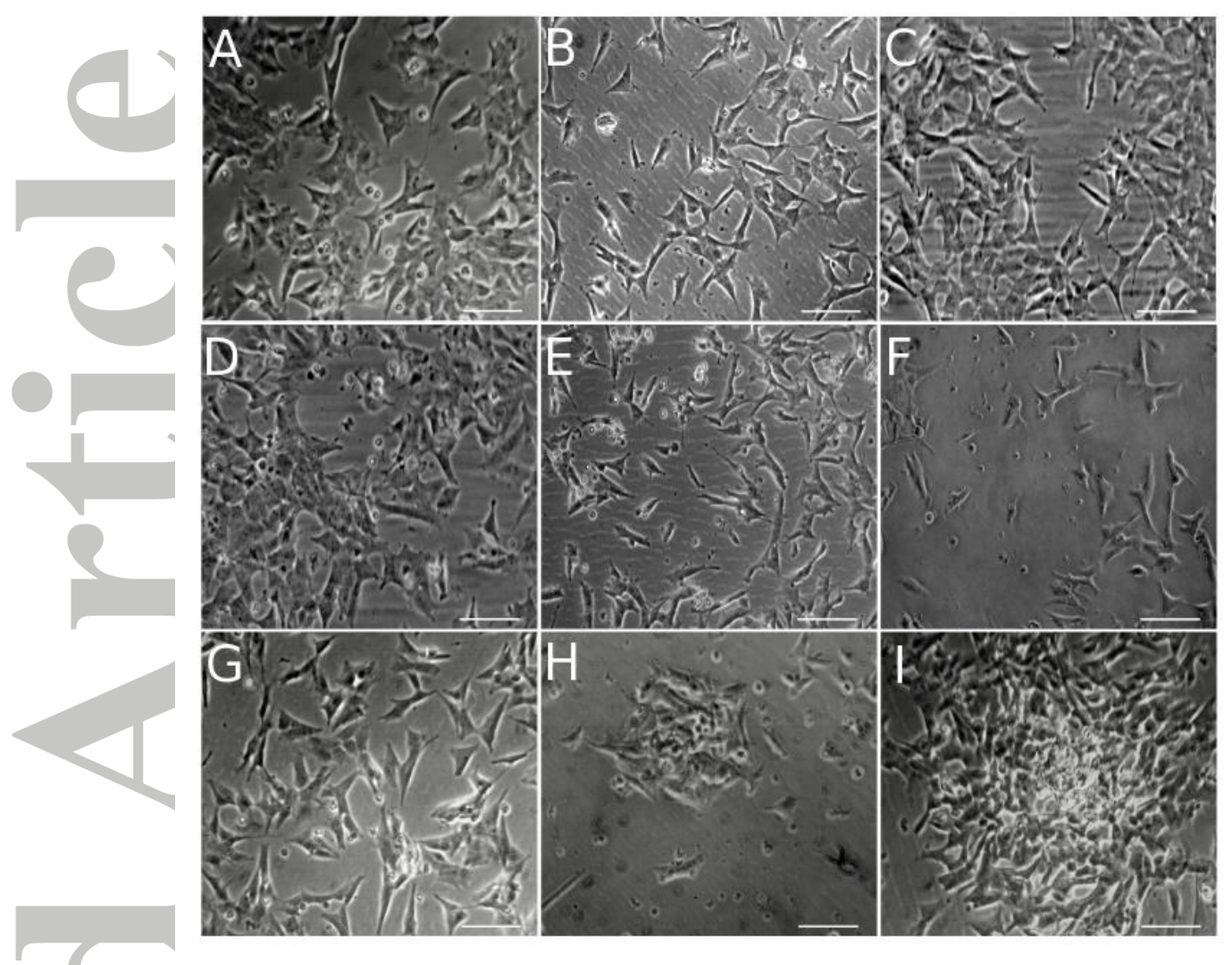

Figure 3. After 3 weeks of encapsulation cells tend to form tight clusters mimicking the behavior in 3D conditions. Brightfield images of morphological changes and growth patterns of SHSY5Y cells encapsulated in $2 \%$ alginate beads by EHDJ method for different times, released and cultured on TCP for 24 hours. Experimental groups were compared to the control, cells cultured without prior encapsulation. A) Control; B) cells encapsulated for 3 hours; C) 1; D) 3; E) 5; F) 7; G) 14; H) 21 and I) 28 days respectively. Scale bar: $50 \mu \mathrm{m}$.

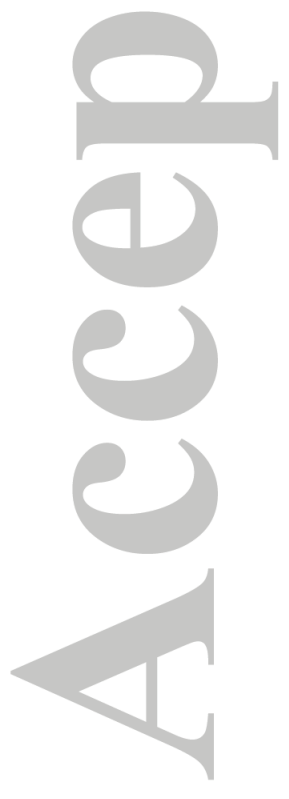




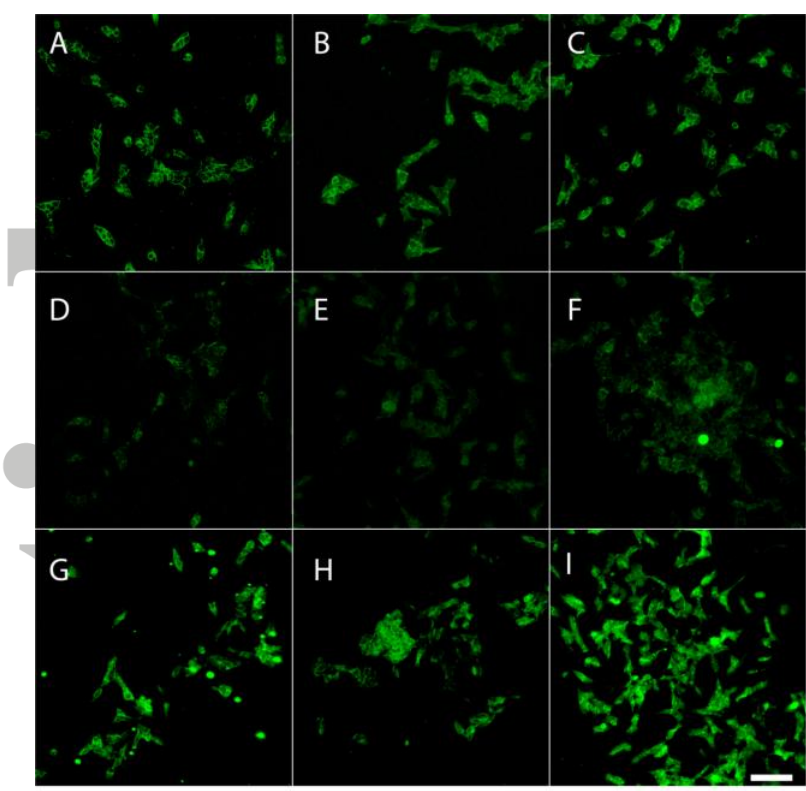

Day 1

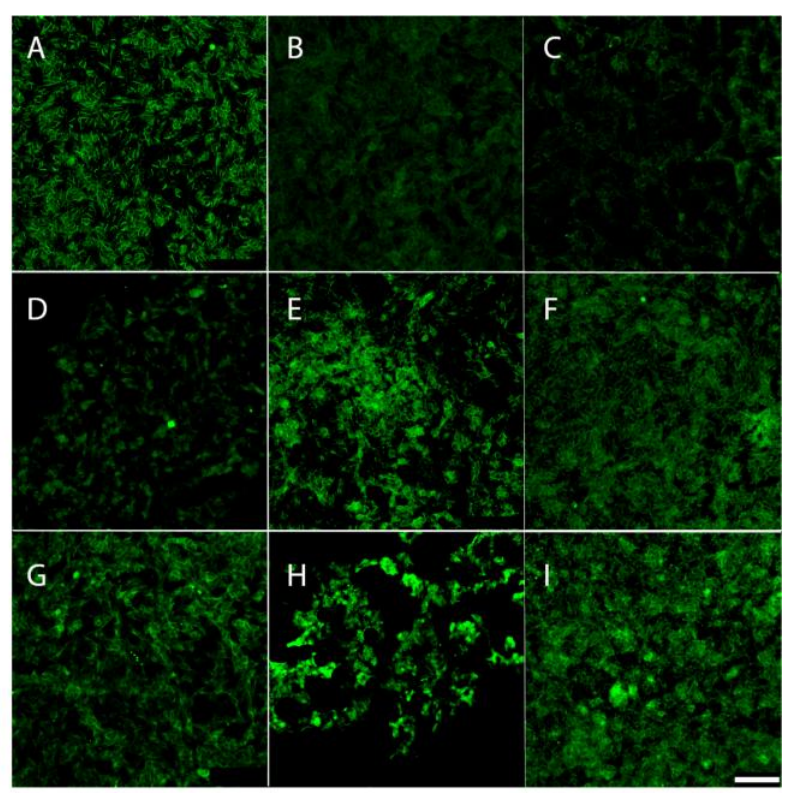

Day 7

Figure 4. Cells undergo epithelial-to-mesenchymal transition 3 days after encapsulation. Immunocytochemistry against $\mathrm{N}$-cadherin evaluated 1 and 7 days after reseeding. Cells were reseeded at 8 experimental time points and compared to the control group of not encapsulated cells grown on TCP. A) Control; B) cells encapsulated for 3 hours; C) 1, D) 3, E) 5, F) 7, G) 14, H) 21 and I) 28 days respectively. Scale bar: $50 \mu \mathrm{m}$.

This article is protected by copyright. All rights reserved. 


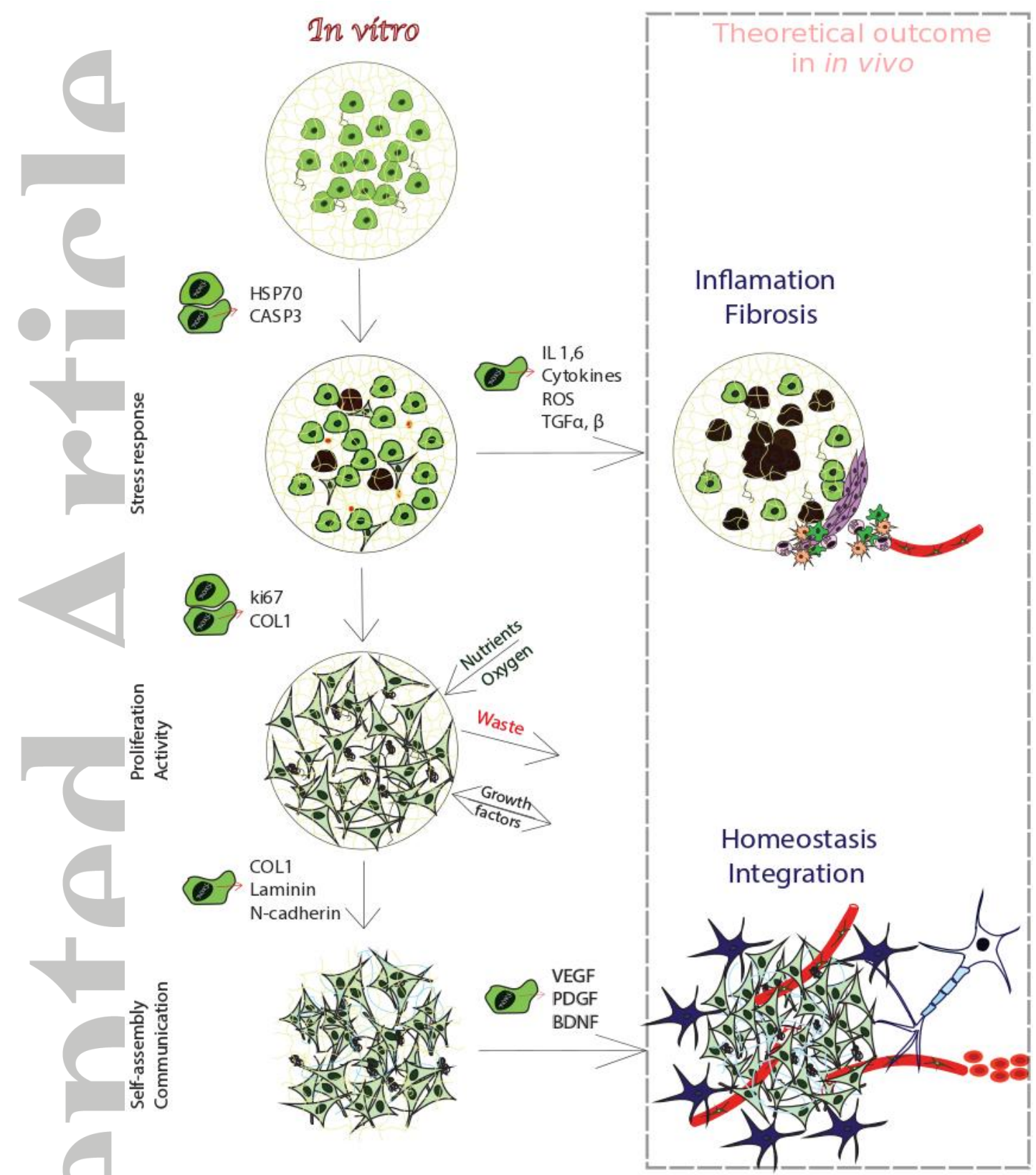

Figure 5. Requirements for successful integration of the engineered tissue in in vivo. Theoretical scheme of the necessary maturation steps for engineered tissue to be successfully integrated into the native environment. ${ }^{1,36,37}$ 